\title{
Persistent memories in transient networks
}

\author{
Andrey Babichev ${ }^{1,2}$ and Yuri Dabaghian ${ }^{1,2 *}$ \\ ${ }^{1}$ Jan and Dan Duncan Neurological Research Institute, \\ Baylor College of Medicine, Houston, TX 77030, \\ ${ }^{2}$ Department of Computational and Applied Mathematics, \\ Rice University, Houston, TX 77005 \\ *e-mail: dabaghia@bcm.edu
}

(Dated: September 16, 2018)

Spatial awareness in mammals is based on an internalized representation of the environment, encoded by large networks of spiking neurons. While such representations can last for a long time, the underlying neuronal network is transient: neuronal cells die every day, synaptic connections appear and disappear, the networks constantly change their architecture due to various forms of synaptic and structural plasticity. How can a network with a dynamic architecture encode a stable map of space? We address this question using a physiological model of a "flickering" neuronal network and demonstrate that it can maintain a robust topological representation of space. 


\section{INTRODUCTION}

It is believed that mammalian ability to navigate, to escape from predators, to find its nest, to plan hunting strategies and so forth, is based on an internalized neuronal representation of space-a cognitive map of the environment [1, 3]. Neurophysiologically, this map is encoded via timed sequences of quick electrical discharges of the neurons-neuronal spikes-in various parts of the brain. A particularly important role in producing this map is played by the hippocampus - one of the oldest parts of the mammalian brain in evolutionary terms. The spike times of the hippocampal principal neurons-so called place cells-are defined by the animal's spatial location: in rodents, each place cell fires when the animal visits a specific location-this cell's place field [3] (Fig. 14A). Why a given place cell fires only when an animal is "here" rather than "there" is still a mystery, and how the ensemble of place cells forms a hippocampal map of the environment is equally mysterious. In particular, it remains largely unknown how the spike trains produced by the place cells are processed downstream from the hippocampus.

Experimental evidence points out that groups of coactive place cells form functionally interconnected "assemblies" [4, 5] that together drive their respective "read-classifier" or "readout" neurons in the downstream networks (Fig. 1B). Since coactivity of the place cells marks the overlap of their respective place fields (Fig. 1C), the activity of a readout neuron actualizes a connectivity relationship between the regions encoded by the individual place cells. This suggests that the hippocampus provides a qualitative representation of space, based on connectivity, adjacency and containment relationships, i.e., that the hippocampal cognitive map is topological in nature-a hypothesis that receives an increasing amount of experimental support[6-10].

In [11, 12] we proposed a theoretical model that showed that hippocampal cell assembly network can indeed capture the spatial connectivity of the environment in a biologically plausible time, given that the place cells operate within biological parameters of firing rate and place field size. However, the approach of [11, 12] was based on analyses of the ever-growing pool of spike trains, i.e., it ignored that, in physiological networks, the connectivity information may not only accumulate, but also decay. In particular, the physiological cell assemblies may not only form, but also disband as a result of deterioration of synaptic connections [13], then reappear as a result of subsequent learning, then disband again and so forth. Electrophysiological studies suggest that the lifetime of the cell assemblies ranges between tens of milliseconds to minutes or longer [4, 5, 14, 15], whereas spatial memories in rats can last for days and months [16-18]. How can the large-scale spatial representation of the environment be stable if the neuronal stratum that computes this representation changes on a much faster timescale? Below we discuss a model of a transient hippocampal network and use methods of Algebraic Topology to demonstrate that the topological characteristics of the large-scale spatial representation of the environment encoded by this network can remain stable.

\section{MODEL}

The way place fields cover an environment $\mathcal{E}$ (Fig. 11A) calls to mind a basic theorem of algebraic topology due to P. Alexandrov [19] and E. Čech [20]: it is possible to reconstruct the topology of a space $X$ from the pattern of overlaps between the regions that cover it. The model proposed in [11, 12, 21] is based on the observation that this theorem can not only be applied to the place fields themselves, but also implemented via spiking signals of cells that represent these place fields. In this approach, groups of coactive place cells, $c_{1}, c_{2}, \ldots, c_{n}$, are viewed as abstract simplexes, $\sigma=\left[c_{1}, c_{2}, \ldots, c_{n}\right]$, which together form a simplicial "cell assembly complex," $\mathcal{T}_{C A}$ (for definitions and details see [22, 23] and Methods in [11]).

This construction provides a connection between the local (cellular) and the global (system-level) scales: the individual cell assemblies, just like simplexes, provide local information about the space, but together, as a neuronal ensemble, they represent space as whole - as the simplicial complex. Thus, $\mathcal{T}_{C A}$ provides a schematic representation of the cell assembly network and of its rewiring dynamics: formation of new 
A.

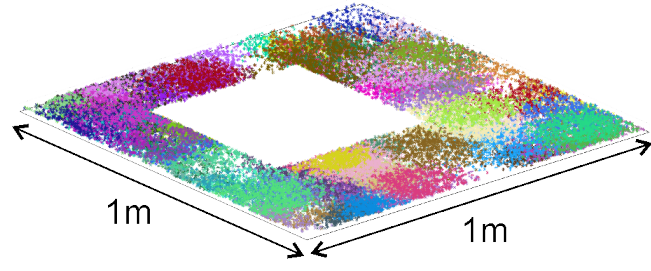

B.

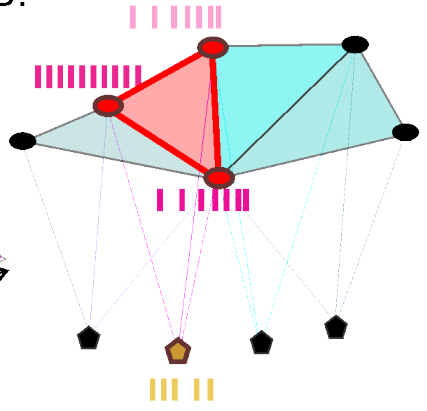

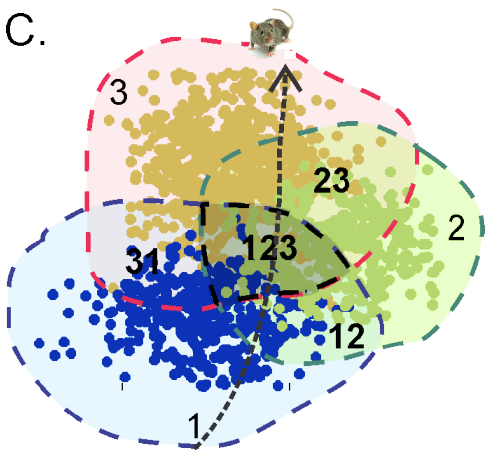

FIG. 1: Place cells and cell assemblies. (A). Simulated place fields in a small planar environment containing a square hole: dots of different color mark the locations where the corresponding place cells produced spikes. The dots of a particular color form spatial clusters - the place fields. (B). Schematic representation of four overlapping third-order cell assemblies by four third order simplexes (triangles). The vertexes (shown by small circles) represent place cells synaptically connected to their respective readout neurons (pentagons). A cell assembly $\sigma$ "ignites" (red triangle) when all of its place cells become simultaneously active, thus eliciting a response from the readout neuron $n_{\sigma}$ (active cells have colored centers). (C). A coactivity of the place cells $c_{1}, c_{2}$ and $c_{3}$ occurs when the rat crosses through the overlap of the corresponding place fields-the domain 123.

place cell assemblies and disbanding of some old ones are represented, respectively, by the appearance and disappearance of their counterpart (maximal) simplexes in $\mathcal{T}_{C A}$.

On the other hand, the cell assembly complex $\mathcal{T}_{C A}$ provides semantics for describing the global spatial memory map in topological terms [24]. For example, a sequence of cell assemblies ignited along a path $\gamma$ navigated by the rat corresponds to a chain of simplexes $\Gamma \in \mathcal{T}_{C A}-\mathrm{a}$ "simplicial path" (Fig. 2). The structure of $\mathcal{T}_{C A}$ allows to establish certain qualitative properties of the simplicial paths and to relate them to the properties of the physical paths in $\mathcal{E}$. For example, a simplicial path that closes onto itself in $\mathcal{T}_{C A}$ may represent a closed physical path, a pair of topologically equivalent simplicial paths $\Gamma_{1} \sim \Gamma_{2}$ may represent physical paths $\gamma_{1}$ and $\gamma_{2}$ that can be deformed into one another, a hole in $\mathcal{T}_{C A}$ may represent a physical obstacle and so forth. However, establishing these correspondences requires learning: as the animal begins to explore the environment, only a few place cells would have time to (co)activate, and only a few cell assemblies would have time to form; as a result, a newly developing complex $\mathcal{T}_{C A}$ consists of only a few maximal simplexes and contains many holes, some of which correspond to physical obstacles or to regions that have not yet been visited by the animal, and others are "spurious", i.e., reflecting transient information about place cell coactivity. As shown in [11, 12], the spurious holes tend to disappear as more spatial information accumulates.

In [25] we suggested two methods for constructing the cell assembly network and hence producing the simplicial cell assembly complex $\mathcal{T}_{C A}$ that represents this network, by selecting the most frequent combinations of spiking place cells. The key observation of [25] was that in bounded environments, the coactive cell combinations eventually become repetitive, and it is therefore possible to identify the cell assemblies from the most frequent coactivity patterns. However, the frequency of a given cell assembly's activation, $f_{\sigma}$, was evaluated from the total number of its appearances over the entire navigation period, and then the selected cell assemblies were presumed to have existed for as long as the navigation continued. In other words, the cell assemblies were "perennial" by construction. In the following, we model a transient hippocampal network by limiting the time during which a cell assembly $\sigma$ can form to a smaller time interval- "a memory window" $W^{(\sigma)}$. Physiologically, $W^{(\sigma)}$ defines the period during which readout neuron $n_{\sigma}$ can identify the combination $\sigma$ of coactive place cells, connect to it synaptically, retain these connections and respond to subsequent ignitions of $\sigma$.

To simplify the approach, we consider the case in which the entire ensemble of readout neurons is characterized by a single parameter $W^{(\sigma)}=W$, and proceed as follows. First, we identify the cell assemblies 

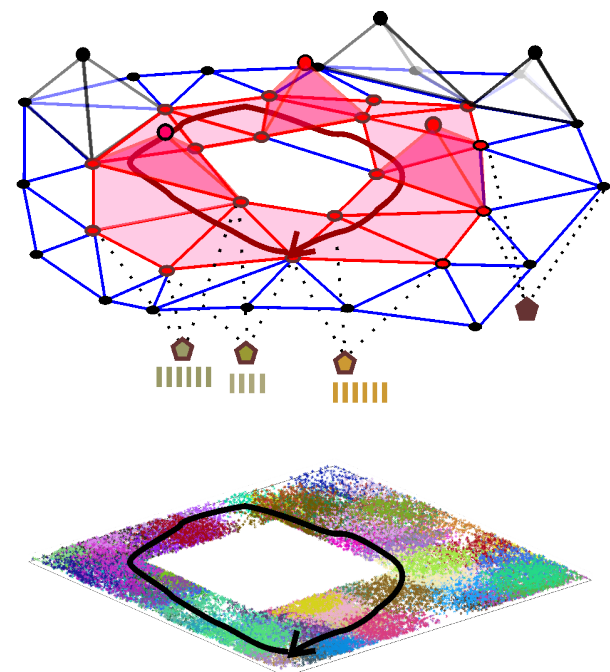

FIG. 2: Scehamtic representation of place cell coactivity in the cell assembly complex $\mathcal{T}_{C A}$. The topological structure of $\mathcal{T}_{C A}$, induced from the place field layout in $\mathcal{E}$, provides a topological representation of $\mathcal{E}$. For example, the hole in the middle of $\mathcal{T}_{C A}$, which produces non-contractible simplicial paths (topological loops) corresponds to the central hole in the environment $\mathcal{E}$, which produces non-contractible navigation paths $\gamma$ (e.g., a sample path is shown at the bottom). As the animal travels along $\gamma$, the hippocampal place cell assemblies ignite in a certain order, which corresponds to a chain of maximal simplexes in $\mathcal{T}_{C A}$-a simplicial path $\Gamma$, shown by red triangles and tetrahedrons.

that emerge within the first $W$-period after the onset of the navigation, $W_{1}$, and then repeat the algorithm for the subsequent windows $W_{2}, W_{3}, \ldots$, obtained by shifting the $W_{1}$ over small time steps. As a result, a cell assembly $\sigma$ that was identified in the window $W_{t_{1}}$, based on the local place cell coactivity rate $f_{\sigma}\left(W_{t_{1}}\right)$, may disappear at a certain step $W_{t_{2}}$, then reappear in a later window, $W_{t_{3}}$, disappear again and so forth. The ensemble of appearing and disappearing cell assemblies can then be schematically represented by a "flickering" simplicial complex, $\mathcal{F}_{W}$, with appearing and disappearing maximal simplexes. Our task is to study the net topological properties of $\mathcal{F}_{W}$, e.g., whether the lifetimes of its topological loops can be longer than the lifetimes of its typical maximal simplex.

\section{RESULTS}

We studied the dynamics of the flickering complex $\mathcal{F}_{W}$ and the topological information encoded in it, as a function of the discrete time $t_{n}=n \Delta t, \Delta t=2.5 \mathrm{secs}$, for the memory window width $W=5$ minutes. The results produced by a typical neuronal ensemble containing $N_{c}=300$ simulated place cells are shown on Fig. 3 First, we observe that the fluctuations of the number of the maximal simplexes in $\mathcal{F}_{W}, N_{\sigma}$, remain confined within about $20 \%$ from the mean, $220 \leq N_{\sigma} \leq 300$, so that the simulated hippocampal network contains about 260 fluctuating cell assemblies on average (Fig. 3A). However, while the size of the flickering complex remains bounded, the pool of the maximal simplexes changes significantly: as temporal separation $\Delta_{i j}=\left|t_{i}-t_{j}\right|$ between the memory windows increases, the corresponding complexes $\mathcal{F}_{W}\left(t_{i}\right)$ and $\mathcal{F}_{W}\left(t_{j}\right)$ become more and more dissimilar (Fig. 3B). After about 50 time steps ( 120 seconds), the set of simplexes in $\mathcal{F}_{W}$ is essentially renewed, which implies that the cell assembly network, as described by the model, is completely rewired (Fig. 3 C).

A typical maximal simplex lasts on average about 10 discrete time steps ( $\tau_{\sigma} \approx 25$ seconds), which is close to the range of values established experimentally [5]. Such rapid rate of the simplex renewal in the flickering cell assembly complex allows us to address our main question: what is the dynamics of the topological characteristics of $\mathcal{F}_{W}$ and how do they correspond to the topology of the environment?

The topology of the environment can be described in terms of the Betti numbers-roughly speaking, a Betti number $b_{n}$ defines the number of $n$-dimensional topological loops in $\mathcal{F}_{W}$ (i.e., closed surfaces counted up to topological equivalence). In the case of the environment shown on Fig. 1 A the Betti numbers are as follows: $b_{0}=1$ (i.e., the environment is connected), $b_{1}=1$ (i.e., there is one $1 D$ topological loop that encircles the hole in the middle), while $b_{n>1}=0$ (no topological loops in higher dimensions). Using the methods of Persistent Homology [26-28], we evaluated these numbers for the flickering cell assembly 
A.

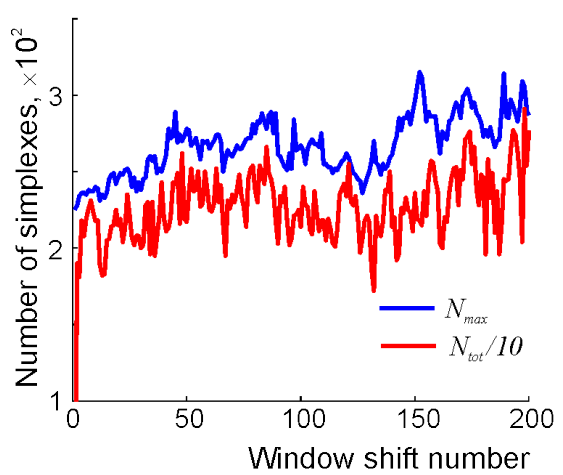

B.

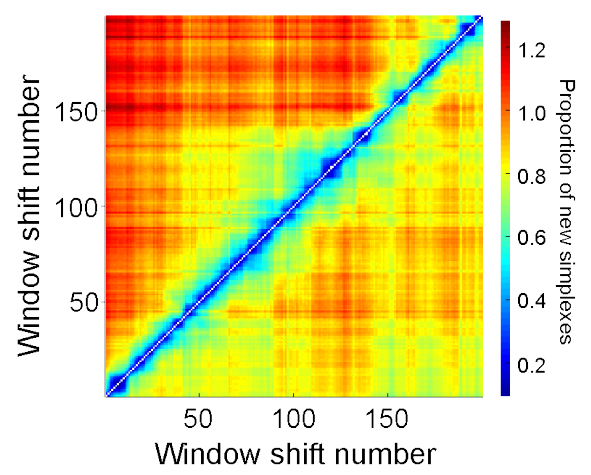

C.

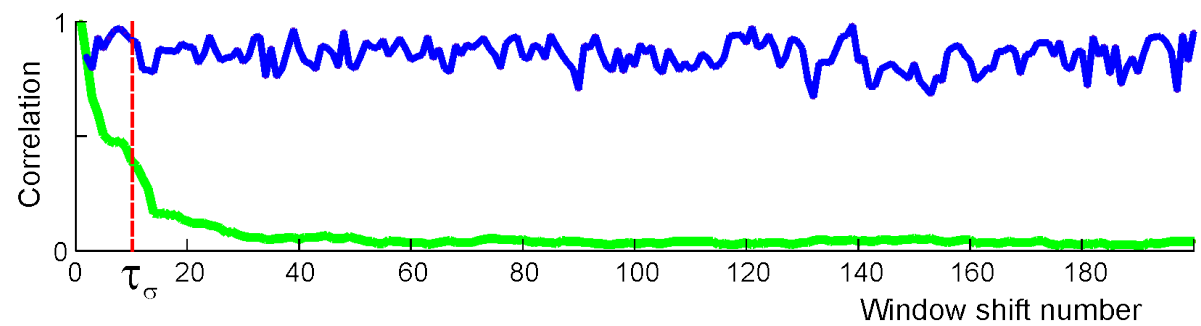

FIG. 3: Time evolution of the cell assembly complex. (A) The number of maximal simplexes (blue trace) and the total number of all simplexes, scaled down by a factor of 10 (red) in the flickering simplicial complex $\mathcal{F}_{W}$. The memory window spans over $W=5$ minutes, shifting over $\Delta t=2.5$ secs at a time. Shown are first 200 shifts. (B) The matrix of similarity coefficients, $r_{i j}$, between the pairs of flickering cell assembly complexes $\mathcal{F}_{W}\left(t_{i}\right)$ and $\mathcal{F}_{W}\left(t_{j}\right)$ defined as the proportion of the maximal simplexes contained in $\mathcal{F}_{W}\left(t_{i}\right)$ but not in $\mathcal{F}_{W}\left(t_{j}\right)$. For close moments $t_{i}$ and $t_{j}$, the differences are small, but as time separation grows, the differences becomes larger. (C) At every moment of time $t_{i}$, the blue line shows the fraction of the maximal simplexes that were also present at the previous moment, $t_{i-1}$. The green line shows the fraction of the original maximal simplexes (from $\mathcal{F}_{W}\left(t_{1}\right)$ ) remaining in $\mathcal{F}_{W}\left(t_{i}\right)$. The population of simplexes changes entirely (by about $0.95 \%$ ) in about 60 steps. The dashed line marks the mean simplex decay rate, $\tau_{\sigma}=10$ window shifts (about 25 seconds).

complex for the sequence of windows, and found that $\mathcal{F}_{W}$ does, in fact, reliably capture the topological properties of the environment over long periods, which significantly exceed both the simplexes' lifetimes, $\tau_{\sigma}$, and the width of the memory window, $W$.

As illustrated on Fig. 4A, the first and the second Betti numbers almost never deviate from their respective physical values $\left(b_{1}=1\right.$ and $\left.b_{2}=0\right)$. The occasional changes of $b_{1}$ and $b_{2}$ can be viewed as short-time "topological fluctuations" in the hippocampal map. For example, an occurrence of $b_{1}=2$ value indicates the appearance of an extra (non-physical) $1 D$ loop, and at the moments when $b_{1}=0$, all $1 D$ loops in $\mathcal{F}_{W}$ are contractible, i.e., the central hole (Fig. 1A) is not represented in the hippocampal map. Similarly, the moments when $b_{2} \neq 0$ indicate times when the flickering complex $\mathcal{F}_{W}$ contains non-physical, non-contractible $2 D$ bubbles (one can speculate about the cognitive effects that these fluctuations may produce). The 0-th and the third Betti number always came out correct, $b_{0}=1, b_{3}=0$, implying that despite the fluctuations of its simplexes, the cell assembly complex $\mathcal{F}_{W}$ does not disintegrate into pieces and remains contractible in higher $(D>2)$ dimensions, i.e., that the topological fluctuations in the hippocampal map are bounded to $1 D$ paths and $2 D$ surfaces.

Further numerical analyses demonstrate that, as the memory window increases, the Betti numbers $b_{1}$ and $b_{2}$ become more stable. In contrast, as the memory window shrinks, the fluctuations of the topological loops intensify (Fig. 4B). This implies that if the characteristic timescale of the network's transience is sufficiently large, then the corresponding coactivity complex remains fixed and topologically equivalent to the "perennial" cell assembly complex, $\mathcal{F}_{W=\infty}=\mathcal{T}_{C A}$. On the other hand, if the simplexes are too unstable, 

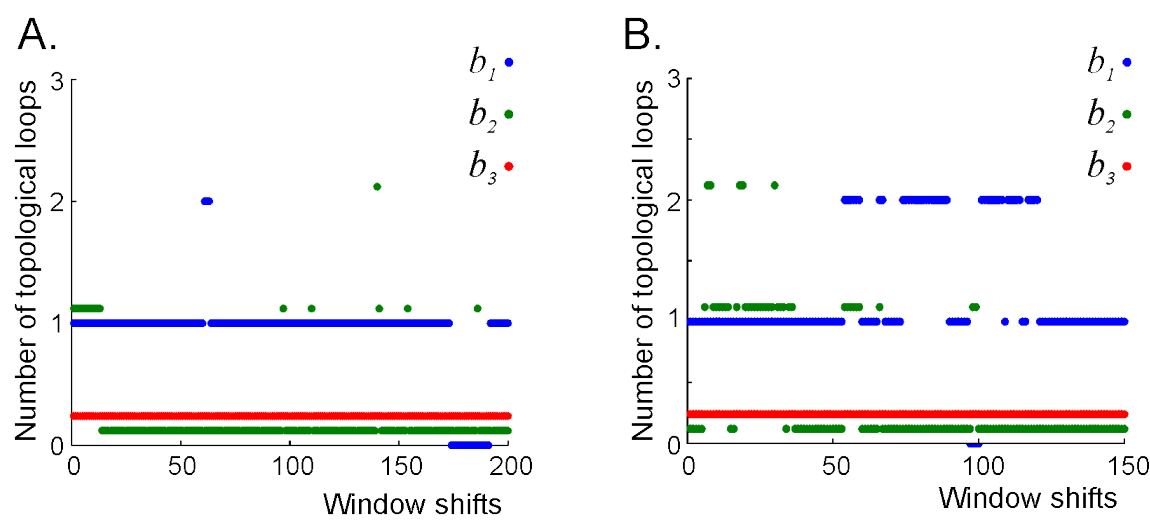

FIG. 4: Stability of the topological information. (A). The low-dimensional Betti numbers, $b_{1}, b_{2}, b_{3}$ as a function of the discrete time, computed using 5 minute wide memory window $W$. The 0th Betti number $\left(b_{0}=1\right.$ at all times) is not shown. (B). As the memory window decreases to $W=3$ minutes, the frequency and the number of the topological fluctuations increases: the flickering complex repeatedly produces an extra (spurious) $1 D$ topological loop and up to two spurious bubbles.

then the cell assembly complex $\mathcal{T}_{C A}$ fails to acquire the correct physical structure, no matter how long is the learning period.

\section{DISCUSSION}

In physical literature, fluctuating simplicial complexes have been previously used in the context of Simplicial Quantum Gravity theories for discretizing quantum fluctuations of the space-time [29, 30]. A natural requirement for these theories is that the quantum fluctuations at the micro-scale should average out in the thermodynamic limit, yielding a smooth space-time continuum at the macroscale.

There are certain parallels with cognitive representations of space that emerge from the spiking activity of neuronal ensembles. At the micro level, the encoded spatial information is naturally discrete at the cellular level and allows a schematic representation in terms of simplicial complexes [11, 12, 24, 25]. Moreover, since the various place cell assemblies that spike at different locations are transient structures, these auxiliary simplicial complexes fluctuate. On the macro level, the emergent hippocampal maps provide a stable topological representation of the physical environment over long periods [10, 16-18], enabling topological reasoning during animals' spatial navigation [8, 9].

Our model provides one explanation for how these two experimentally established aspects of hippocampal neurophysiology can coexist. According to the model, while the simplexes of the flickering simplicial complex $\mathcal{F}_{W}$ fluctuate at the physiological timescale, its Betti numbers can keep their physical values indefinitely. The fact that the simplex fluctuations can average out at the scale of the entire complex suggests that rapid rewiring of the cell assemblies can preserve the stability of the cognitive map as a whole.

Although these results were obtained using a simple computational model, we hypothesize that the observed effect reflects a more general phenomenon that might apply to physiological mechanisms of synaptic and structural plasticity in the hippocampal network.

\section{METHODS}

Place cell spiking activity is modeled as a stationary temporal Poisson process with a spatially localized rate [31] that is characterized by the spatial location of the peak, $r_{c}$, the peak firing amplitude, $f_{c}$, and the place field size, $s_{c}$. We simulated an ensemble of $N_{c}=300$ place cells with log-normally distributed peak 
firing amplitudes, with the mean $\left\langle f_{c}\right\rangle=14 \mathrm{~Hz}$, log-normally distributed place field sizes with the mean $\left\langle s_{c}\right\rangle=17 \mathrm{~cm}$ (for details see Methods in [11]). Spiking is modulated by the $8 \mathrm{~Hz} \theta$-oscillations-the basic rhythm of the extracellular field in the hippocampus [32]. Neuronal coactivity is defined as firing that occurred over two consecutive $\theta$ cycles—a computationally established optimal value [12], which corresponds to the parameters estimated from experimental data [32, 33]. The topological analyses were implemented using the JPlex package [34].

\section{ACKNOWLEDGMENTS}

The work was supported by the NSF 1422438 grant and by Houston Bioinformatics Endowment Fund.

\section{REFERENCES}

[1] O'Keefe J, Nadel L (1978) The hippocampus as a cognitive map, New York: Clarendon Press; Oxford University Press. xiv, 570 pp.

[2] Tolman EC (1948) Cognitive maps in rats and men, Psychol. Rev. 55: 189-208.

[3] Tolman PJ, White AM, Minai A (2001) Spatial processing in the brain: the activity of hippocampal place cells, Ann. Rev. Neurosci., 24: 459-486.

[4] Harris KD, Csicsvari J, Hirase H, Dragoi G, Buzsaki G (2003) Organization of cell assemblies in the hippocampus, Nature 424: 552-556.

[5] Buzsaki G (2010) Neural syntax: cell assemblies, synapsembles, and readers, Neuron 68: 362-385.

[6] Gothard KM, Skaggs WE, McNaughton BL (1996) Dynamics of mismatch correction in the hippocampal ensemble code for space: interaction between path integration and environmental cues, J. Neurosci, 16: 8027-8040.

[7] Leutgeb JK, Leutgeb S, Treves A, Meyer R, Barnes CA, et al. (2005) Progressive transformation of hippocampal neuronal representations in "morphed" environments, Neuron 48: 345-358.

[8] Alvernhe A, Sargolini F, Poucet B (2012) Rats build and update topological representations through exploration, Anim. Cogn. 15: 359-368.

[9] Poucet B, Herrmann T (2001) Exploratory patterns of rats on a complex maze provide evidence for topological coding, Behav. Processes, 53, pp. 155-162.

[10] Dabaghian Y, Brandt VL, Frank LM (2014) Reconceiving the hippocampal map as a topological template, eLife 10.7554/eLife.03476.

[11] Dabaghian Y, Mmoli F, Frank L, Carlsson G (2012) A Topological Paradigm for Hippocampal Spatial Map Formation Using Persistent Homology, PLoS. Comput. Biol. 8: e1002581.

[12] Arai M, Brandt V, Dabaghian Y (2014) The Effects of Theta Precession on Spatial Learning and Simplicial Complex Dynamics in a Topological Model of the Hippocampal Spatial Map. PLoS Comput. Biol. 10: e1003651.

[13] Wang Y, Markram H, Goodman PH, Berger TK, Ma J, et al. (2006) Heterogeneity in the pyramidal network of the medial prefrontal cortex, Nat. Neurosci. 9: 534-542.

[14] Bi G-q, Poo M-m (2001) Synaptic Modification by Correlated Activity: Hebb's Postulate Revisited, Ann. Rev. Neurosci. 24: 139-166.

[15] Magee JC, Johnston D (1997) A Synaptically Controlled, Associative Signal for Hebbian Plasticity in Hippocampal Neurons, Science 275: 209-213.

[16] Meck WH, Church RM, Olton DS (2013) Hippocampus, time, and memory, Behav. Neurosci., 127: 655-668.

[17] Clayton NS, Bussey TJ, Dickinson A (2003) Can animals recall the past and plan for the future?, Nat. Rev. Neurosci., 4: 685-691.

[18] Brown MF, Farley RF, Lorek EJ (2007) Remembrance of places you passed: Social spatial working memory in rats, Journal of Experimental Psychology, 33: 213-224.

[19] Alexandroff P, (1928) Untersuchungen Uber Gestalt und Lage Abgeschlossener Mengen Beliebiger Dimension. Annals of Mathematics 30: 101-187. 
[20] Čech E (1932) Theorie generale de lhomologie dans une space quelconque, Fundamenta mathematicae 19: 149-183.

[21] Curto C, Itskov V (2008) Cell groups reveal structure of stimulus space, PLoS Comput. Biol. 4: e1000205.

[22] Hatcher A (2002) Algebraic topology, Cambridge; New York: Cambridge University Press.

[23] Aleksandrov P (1965) Elementary concepts of topology, New York: F. Ungar Pub. Co. 63 pp.

[24] Babichev A, Cheng S, Dabaghian Y (2015) Topological schemas of cognitive maps and spatial learning in the hippocampus, in submition; arXiv:1509.00171.

[25] Babichev A, Memoli F, Ji D, Dabaghian Y (2015) Combinatorics of Place Cell Coactivity and Hippocampal Maps. in submition; arXiv:1509.01677

[26] Ghrist R (2008) Barcodes: The persistent topology of data, Bulletin of the American Mathematical Society 45: 61-75.

[27] Zomorodian AJ (2005) Topology for computing, Cambridge, UK ; New York: Cambridge University Press. xiii, $243 \mathrm{pp}$.

[28] Edelsbrunner H, Harer J (2010) Computational topology: an introduction, Providence, R.I.: American Mathematical Society. xii, $241 \mathrm{pp}$.

[29] Ambjørn J, Carfora M, Marzuoli A (1997) The geometry of dynamical triangulations, Berlin ; New York: Springer. viii, $197 \mathrm{pp}$.

[30] Hamber HW (2009) Quantum gravitation: the Feynman path integral approach, Berlin: Springer. xvii, 342 pp. Nuclear Physics B - Proceedings Supplements 94: 689-692.

[31] Barbieri R, Frank LM, Nguyen DP, Quirk MC, Solo V, et al. (2004) Dynamic analyses of information encoding in neural ensembles, Neural Comput. 16: 277-307.

[32] Buzsaki G (2002) Theta oscillations in the hippocampus, Neuron 33: 325-340.

[33] Buzsaki G (2005) Theta rhythm of navigation: link between path integration and landmark navigation, episodic and semantic memory, Hippocampus 15: 827-840.

[34] JPlex freeware was developed by ComTop group, Stanford University. 